\title{
CLINICAL OUTCOME OF AN INJECTABLE PLATELET-RICH FIBRIN AND PLATELET-RICH PLASMA ON IMMEDIATE DENTAL IMPLANT
}

\author{
Haitham Abdel Karim Ahmed El Komi ${ }^{* *}$, Mohamed Mohamed Bayomi ${ }^{2}$, Mahmoud Taha Eldestawy ${ }^{3}$
}

\begin{abstract}
Objective: The aim of the present study was to evaluate the implant stability with injectable-PRF and with PRP on immediate implant. Subjects \& Methods: Twenty patients were selected: ten patients with immediate implant were treated with I-PRF. PRP group: ten patients with immediate implant were treated with PRP. Clinical evaluation was recorded after 0,3 and 6 months after surgery: Modified plaque index (mPII), Probing pocket depth around implants, and Implant stability. Results: In The present study, i- PRF application during implant surgery enhanced the stability of implants than PRP. Although the sample size was limited, this technique provided an advantage, especially when bone quality at the implant site was inadequate. Conclusion: The results of the present study demonstrate that the application of injectable platelet rich fibrin during implant surgery enhanced the stability of implants when compared to platelet rich plasma.
\end{abstract}

KEYWORDS: Immediate dental implant, Injectable platelet-rich fibrin, Platelet-rich plasma, implant stability.

\section{INTRODUCTION}

More than 30 years of clinical experience and scientific background have clearly demonstrated that oral rehabilitation with implant-supported prostheses is a safe method with high success rates $^{(1,2)}$. The original protocol included the implant is submerged positions for a few months to avoid occlusal load and contamination from the oral cavity during the healing period. Further study have shown that the osseointegration process occurs even in a trans-mucosal environment with the same rate of success, ${ }^{(3)}$ Implant installation immediately after tooth extraction has been investigated in several clinical and histologic studies and today there is scientific evidence showing that osseointegration may be achieved even at implants placed in a fresh extraction socket ${ }^{(4-7)}$.

The placement of implants in fresh extraction sockets allows placement of the implant during the same visit at which the tooth is extracted, which reduces morbidity; decreases the treatment time by reducing the surgical procedures, thus improving the psychologic approach with the patient; and may achieve an optimal esthetic result. Furthermore, placement of an implant immediately after tooth extraction may help maintain the bone crest and

1. Masters Candidate, Department of Oral Medicine, Faculty of Dental Medicine, Boys, Al-Azhar University, Cairo.

2. Professor of Oral Medicine Periodontology, Oral Diagnosis and Radiology Department, Faculty of Dental Medicine (Boys, Cairo) Al-Azhar University

3. Ass. Professor of Oral Medicine Periodontology, Oral Diagnosis and Radiology Department. Faculty of Dental Medicine (Boys, Cairo) Al-Azhar University

-Corresponding author: haitham_elkomi@hotmail.com 
leads to ideal implant positioning from a prosthetic point of view ${ }^{(8,9)}$. Selection of ideal implant site is the first step in achieving functionally and esthetically successful implant prosthesis.

There is growing scientific evidence showing that osseointegration can be achieved even at implants placed in fresh extraction sockets. ${ }^{(10)}$ When there is a bone defect in the area of implant placement, it has been shown that a combination of bone graft and membrane have resulted in better quality and quantity of regenerated bone than that expected from either a bone graft or a membrane alone ${ }^{(10)}$.

Platelet rich plasma (PRP) is defined as a high concentration of autologous platelets in a small volume of autologous plasma ${ }^{(11,12)}$. Specifically, PRP is a platelet concentration with at least $1,000,000 / 1 \mathrm{~L}$ in a $5 \mathrm{~mL}$ volume of plasma, when normal human platelet counts in the blood range from $150,000 / 1 \mathrm{~L}$ to $350,000 / 1 \mathrm{~L}$. The platelets contained in this concentrate of autologous plasma release their alpha granules after the coagulation process has been locally trigged in the wound site. These alpha granules contain a cocktail of growth factors which promote proliferation, chemotaxis and the differentiation of cells, which are essential to osteogenesis. Thus, besides its procoagulant effect, PRP is a source of growth factors involved in initiating and sustaining wound healing by accelerating bone repair, promoting fibroblast proliferation, and increasing tissue vascularity ${ }^{(13)}$.

Platelet-rich plasma gel is formed by mixing PRP (derived from the centrifugation of autologous whole blood) with thrombin and calcium chloride. Adding thrombin and calcium chloride to PRP automatically activates the alpha granules to release the following biological growth factors: PDGF, TGF-b, VEGF, insulin- like growth factor I, EGF and epithelial cell growth factor ${ }^{(14)}$.

Recently, an injectable formulation of PRF (I-PRF) has been developed without having to use anti-coagulants; I-PRF is formulated by centrifugation at lower speeds $(700 \mathrm{rpm}, 60 \mathrm{~g})$ for only $3 \mathrm{~min}$ and thus must be utilized within $15 \mathrm{~min}$ prior to fibrin clot formation ${ }^{(15)}$. Choukroun and Ghanaati, demonstrated that I-PRF contains a higher proportion of cells including leukocytes prior to the formation of a fibrin clot when compared to other platelet concentrates due to the low centrifugation speeds ${ }^{(16)}$. While leukocytes are not found in every formulation of platelet concentrates, they are immune cells having shown substantial importance during the host-defense response to incoming pathogens, as well as assisting in the wound healing process by secreting various growth factors ${ }^{(17)}$.

Diagnostic imaging plays an important role in evaluating the dental implant patient. These examinations quantify accurate bone height, width \& contour and provide information about the locations of vital anatomic structure adjacent to the sites of implant placement ${ }^{(18)}$. In implant dentistry, threedimensional (3D) imaging can be realized by dental cone beam computed tomography (CBCT), offering volumetric data on jaw bones and teeth with relatively low radiation doses and costs. CBCT has a reasonably low radiation dose and a high spatial resolution $^{(19)}$.

\section{SUBJECTS AND METHODS}

Twenty patients were selected from the outpatient clinic and classified to two groups screened for eligibility to participate in the present clinical trial. I-PRF group: ten patients with immediate implant were treated with I-PRF. PRP group: ten patients with immediate implant were treated with PRP. Age range was from 18 to 43 years were included in this study. The patients were selected on the basis of inclusion and exclusion criteria.

Inclusion criteria include: patients' being free from any systemic disease and at least 18 years old of both sexes, presence of non-restorable maxillary anterior and premolar teeth associated with the buccal bone defect. Exclusion criteria include: patients with parafunctional habits or abnormal ridge relationships, those taking head and neck radiation treatment, smokers, pregnant or lactating females, and those with unacceptable oral hygiene 
Initial therapy: The initial therapy consisted of prophylaxis, supra, and subgingival scaling, subgingival debridement if needed, adjustment of fault restoration and polishing. CBCT images were obtained for all patients before surgery.

\section{Presurgical preparation of patient:}

1. I-PRF Preparation: blood samples were taken with a 24-gauge butterfly needle from the patient's antecubital vein. Samples were collected in 9-mL glass-coated plastic tubes without anti-clotting agent and immediately centrifuged at lower speeds (700 rpm, 60g) for only $3 \mathrm{~min}$ and thus must be utilized within 15 min prior to fibrin clot formation.

2. PRP preparation: blood samples were taken with a 24-gauge butterfly needle from the patient's antecubital vein. Sample was collected in $10-\mathrm{mL}$ of whole blood with anticoagulant (ethylemediaminetetraacetic acid, EDTA) was centrifuged at (900 rpm, g) for 5 min to separate PRP and platete poor plasma(PPP)proteins from red blood cells and then centrifuged a second time at (2000 rpm, g) for 15 min to separate PRP from PPP.

\section{Surgical Procedures:}

Each patient was asked to rinse his mouth with $0.2 \%$ chlorhexidine solution. All the surgical proce- dures were performed under local anesthesia and strict aseptic conditions. The atraumatic extraction of the tooth is essential to prevent damage to the buccal bone plate. After the extraction, sockets were curettage and irrigated with saline to remove granulation tissue and residual periodontal ligament. Surgical sites were prepared according to the standard procedures using standard drills, J dent implant system was used in this study. The length, which used ranged from $10 \mathrm{~mm}$ to $13 \mathrm{~mm}$, and diameter ranged from $3.2 \mathrm{~mm}$ to $4.3 \mathrm{~m}$.

The dental implant platform will be position 1.5 $\mathrm{mm}$ apical to the alveolar crest. The I-PRF will apply to one of the dental implant osteotomies. Then, the related dental implant will thoroughly be rinsed with I-PRF liquid (I- PRF group). Control dental implants will place in the adjacent osteotomy with PRP application (PRP group).

\section{Postoperative care and medication:}

Patients were asked to follow oral hygiene instructions, medications were prescribed, and asked not to function over the surgical site for the initial 3 weeks. A soft diet was recommended throughout the remaining healing period. The patients were followed up frequently at 3 and 6 months postoperatively.

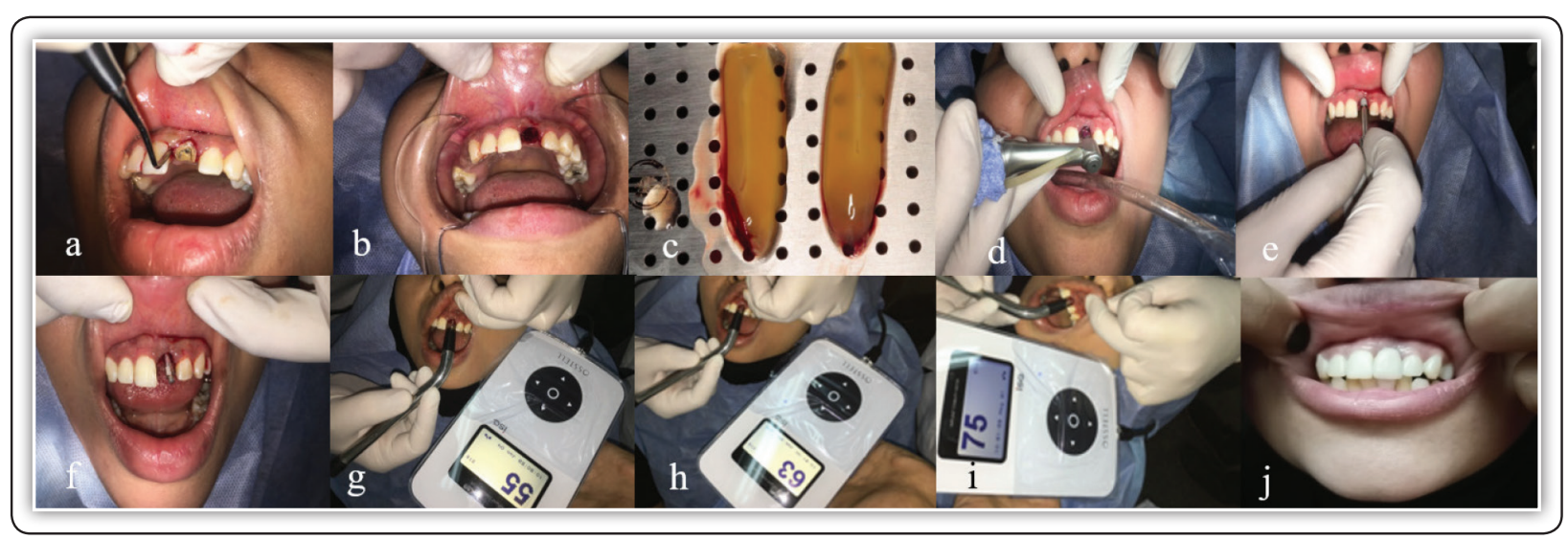

FIG (1) a; Intraoral photograph of atraumatic extraction of tooth (periotome), b; Intraoral photograph after tooth extraction, c; Intraoral photograph after PRP prepared, d \&e; Intraoral photograph showing implant insertion , f; and g; Intraoral photograph showing ostell measurements on the day of surgery, h, Intraoral photograph showing osstell after 3 months, I, Intraoral photograph showing osstell after 6 months, and j, Final restoration. 


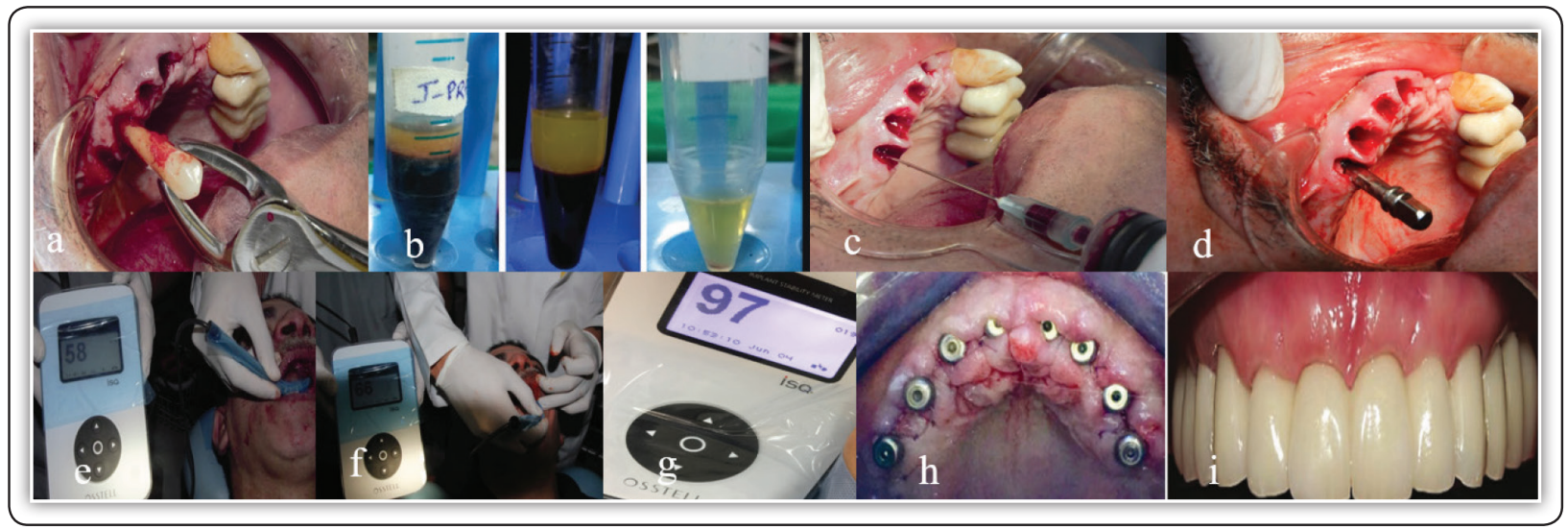

FIG (2) a; Intraoral photograph of atrumatic tooth extraction of tooth, b; Intra oral photograph showing injectable platelet rich fibrin, c; \&d; Intra oral photographs showing insertion of implant and injectable platelet rich fibrin, e; On the surgery day, f, After 3 months, g; After 6 months, h, Intraoral photograph showing complete clearance of upper arch, implants and I-PRF insertion photographs and $\mathrm{j}$, Final restoration.

Clinical evaluation was recorded after 0,3 and 6 months after surgery:

1) Modified plaque index (mPII)

2) Probing pocket depth around implants

3) Implant stability

\section{RESULTS}

In The present study, i- PRF application during implant surgery enhanced the stability of implants than PRP. Although the sample size was limited, this technique provided an advantage, especially when bone quality at the implant site was inadequate.

Table (1) summarizes comparison between the two studied groups according to modified plaque index: at 3 and 6 months, there was a statistically non-significant difference between groups regarding modified plaque index. According to Periimplant pocket depth (PD): at 3 months, there was a statistically non-significant difference between groups regarding Peri-implant pocket depth (PD). At 6 months, there was a statistically a significant difference between groups regarding Peri-implant pocket depth (PD). I-PRF group was lower in Peri-implant pocket depth (PD) than PRP group.
According to stability: at implant placement and 3 months, there was a statistically non-significant difference between groups regarding stability. At 6 months, there was a statistically a significant difference between groups regarding stability, I-PRF group was higher stability than PRP group.

TABLE (1): Summarizes comparison between the two studied groups according to modified plaque index, Peri-implant pocket depth (PD), and Stability.

\begin{tabular}{lccc}
\hline & $\begin{array}{c}\text { PRP } \\
(\mathbf{n}=\mathbf{1 0})\end{array}$ & $\begin{array}{c}\text { I-PRF } \\
(\mathbf{n = 1 0})\end{array}$ & P \\
\hline Modified plaque index & & & \\
\hline 3 months & $0.60 \pm 0.52$ & $0.40 \pm 0.52$ & 0.481 \\
6 months & $0.90 \pm 0.74$ & $0.60 \pm 0.52$ & 0.436 \\
\hline Peri-implant pocket depth $(\mathbf{P D})$ & & \\
\hline 3 months & $1.0 \pm 0.47$ & $0.90 \pm 0.57$ & 0.739 \\
6 months & $2.10 \pm 0.57$ & $1.30 \pm 0.48$ & $0.005^{*}$ \\
\hline Stability & & & \\
\hline At implant placement $(0)$ & $36.60 \pm 2.91$ & $35.70 \pm 3.09$ & 0.511 \\
$3^{\text {th }}$ month (at loading) & $66.30 \pm 9.87$ & $70.50 \pm 11.79$ & 0.399 \\
$6^{\text {th }}$ month & $76.30 \pm 8.14$ & $85.50 \pm 10.02$ & $0.037^{*}$ \\
\hline
\end{tabular}

\section{t: Student t-test}

$p: p$ value for comparing between the studied groups

*: Statistically significant at $p \leq 0.05$ 


\section{DISCUSSION}

Today, the esthetic expectations of patients represent a new challenge in modern implant dentistry. Re-searchers have attempted to accelerate and enhance the process of osseointegration so that patients' functional and esthetic problems can be resolved faster. The aim of the present study was to compare the influence of both PRP and i-PRF on implant stability. While PRP has been investigated in numerous studies as a regenerative blood concentrate, more recently the development of newer platelet formulations with the aim of removing secondary byproducts such as anti-coagulants has been proposed. Much research has shown that PRP releases most of its growth factors at very early time periods whereas PRF induces a more gradual and sustained release of growth factors ${ }^{(20)}$. It has been shown that within the first $10 \mathrm{~min}$, up to $70 \%$ of growth factors are released to the surrounding environment with nearly $100 \%$ released within the first hour ${ }^{(20)}$. These growth factors, despite being released at early time points, have a significant influence on the cellular behavior of many cell types ${ }^{(21-23)}$. With respect to osteoblasts, many studies have previously shown that PRP has a positive influence on either osteoblast proliferation or differentiation ${ }^{(22-25)}$.

PRP hypothesized that the added addition of anti-coagulants has potentially either caused a cytotoxic effect in vivo or significantly reduced the natural coagulation cascade thereby preventing natural wound healing (25-27). For these reasons, recent groups utilizing anticoagulants have thus suggested their removal from their standardized platelet concentrates that have been utilized for over 15 years ${ }^{(17)}$. Therefore, and for these reasons, an increasing trend of utilizing PRF as a replacement blood-derived growth factor for tissue regeneration has been more heavily utilized most noticeably in the dental field ${ }^{(16,28,29)}$. It has been reported that PRF acts to release growth factors much more slowly as a result of both the use of a fibrin scaffold capable of entrapping growth factors and releasing them slowly over time, as well as additionally housing of leukocytes, a cell-type responsible for additional growth factor release ${ }^{(28)}$. Interestingly, two recent articles have further shown that by decreasing centrifugation speeds, a higher proportion of leukocytes could be maintained in the upper layer where PRF is collected and thereby increasing total growth factor release ${ }^{(16,29,30)}$.

The low speed centrifugation concept was utilized to produce a roughly $1 \mathrm{~mL}$ layer of liquid PRF by centrifugation at $700 \mathrm{rpm}$ (only 60G) for 3 min as opposed to conventional PRF centrifugation protocols of $2700 \mathrm{rpm}$ for $12 \mathrm{~min}$. Therefore, while this modified centrifugation protocol produces much less liquid as per not having substantial centrifugation time/speed to separate the two layers, it produces a $1 \mathrm{~mL}$ layer of i-PRF that may be utilized in a liquid formulation prior to fibrin clot formation (coagulation) without having to use anti-coagulants; thereby being $100 \%$ naturallyderived. Therefore, the aim of this study was to first investigate the effect of PRP and i-PRF on human osteoblast behavior. To the best of these authors knowledge, this is the first cell study evaluating the potential of i-PRF on any cell-type.

In the present study, modified plaque index: at 3 and 6 months, there was a statistically nonsignificant difference between groups regarding modified plaque index. At 6 months, there was a statistically a significant difference between groups regarding Peri-implant pocket depth (PD). I-PRF group was lower in Peri-implant pocket depth (PD) than PRP group. i-PRF, an autologous biomaterial rich in growth factors has been shown to improve tissue healing and provides a better understanding of the clinical effects and improvement of clinical parameters.

In the present study, at 6 months, there was a statistically a significant difference between groups regarding stability. I-PRF group was higher stability than PRP group. The results demonstrated that i-PRF application increased the stability of 
implants during the first month of healing. Mean ISQs continuously increased for i- PRF implants, while in the PRP group an increase was seen between the first and fourth weeks. Other studies observed a decrease in stability, mostly during the period between the first and sixth weeks. ${ }^{(31-39)}$. These differences in results may be attributed to either short follow-up periods in the cited studies or to the fact that $\mathrm{i}-\mathrm{PRF}$ accelerates healing. PDGF and PRP were applied around implants to provide bone regeneration and to increase $\mathrm{BIC}{ }^{(31-}$ 37). The histomorphometric findings showed that the amount of bone around the implants exposed to PRP was much greater than that around control implants. However, many studies have stated that growth factors rich in thrombocytes have no significant effects on bone healing ${ }^{(35-38)}$.

A thirty-four implant was placed dental implants in the mandibular interforaminal region and applied PRP to the implant osteotomies on one side. They followed the changes in the stability of the implants with RFA. RFA measurements were made every 4 days until the 44th day after placement. The investigators found statistically non-significant differences between PRP+ and PRP-implants in terms of stability ${ }^{(38)}$. Ergun et al ${ }^{(35)}$ placed implants with PRP and compared their stability with that of a PRP- group over a period of 36 months; no differences were found between groups. They suggested that this could be related to the primary stability of implants. These different results in PRP treatments may be a result of the fact that there is no gold standard preparation of PRP, and various approaches have been used for its preparation.

It has been reported that the biologic effects of PRP seem to start in the second week when PRP with a platelet concentration of approximately $1,000,000 / \mu \mathrm{L}$ is used. In the current study, an effect of i- PRF was detected during the first week ${ }^{(15)}$. This result supports the in vitro findings that showing that i- PRF expresses a stronger and more durable effect on the proliferation and differentiation of osteoblasts than PRP ${ }^{(36-39)}$.
The current study, the mean ISQs for i-PRF implants were $59.39 \pm 15.88$ at the time of insertion and $77.19 \pm 6.06$ at the third week postoperative. On the other hand, the mean ISQs for Prp- implants were $62.67 \pm 13.61$ at the time of insertion and $70.49 \pm 7.74$ at the third week postoperative. These findings are in accordance with previous studies demonstrating that secondary stability increases over time. The present study, i- PRF application during implant surgery enhanced the stability of implants than PRP. Although the sample size was limited, this technique provided an advantage, especially when bone quality at the implant site was inadequate.

\section{CONCLUSION}

The results of the present study demonstrate that the application of injectable platelet rich fibrin during implant surgery enhanced the stability of implants when compared to platelet rich plasma. Within the limitations of this study, the results indicate that i-PRF application results in improved implant stability in the fourth week of osseointegration. However, further clinical, and histologic are required to support these result

\section{REFERENCES}

1. Albrektsson T, Dahl E, Enbom L, Engevall S, Engquist B, Eriksson AR, et al. Osseointegrated oral implants: a Swedish multicenter study of 8139 consecutively inserted Nobelpharma implants. J Periodontol. 1988;59(5):287-96.

2. Bahat $\mathrm{O}$. Brånemark system implants in the posterior maxilla: clinical study of 660 implants followed for 5 to 12 years. Int J Oral Maxillofac Implants. 2000;15(5): 646-53

3. Ericsson L, Randow K, Nilner K, Petersson A. Some clinical and radiographical features of submerged and non-submerged titanium implants. A 5-year follow-up study. Clin Oral Implants Res. 1997;8(5):422-6.

4. Grunder U. Immediate functional loading of immediate implants in edentulous arches: two-year results. Int J Periodontics Restorative Dent. 2001;21(6). 545-51

5. Polizzi G, Grunder U, Goené R, Hatano N, Henry P, Jackson WJ, et al. Immediate and delayed implant placement into extraction sockets: a 5-year report. Clin Implant Dent Relat Res. 2000;2(2):93-9. 
6. Gelb D. Immediate implant surgery: three-year retrospective evaluation of 50 consecutive cases. Int J Oral Maxillofac Implants. 1993;8(4).388-99

7. Barzilay I, Graser N, Iranpour B, Natiella J, Proskin H. Immediate Implantation of Pure Titanium Implants Into Extraction Sockets of Macaca Fascicularis Part II: Histologic Observations. Int J Oral Maxillofac Implants. 1996;11(4).299-310

8. Scarano A, Iezzi G, Petrone G, Marinho V, Corigliano M, Piattelli A. Immediate postextraction implants: a histologic and histometric analysis in monkeys. J Oral Implantol . 2000;26(3):163-9.

9. Crespi R, Capparè P, Gherlone E, Romanos G. Immediate occlusal loading of implants placed in fresh sockets after tooth extraction. Int $\mathrm{J}$ Oral Maxillofac Implants. 2007;22(6).955-62

10. Zitzmann N, Naef R, Schärer P. Resorbable versus nonresorbable membranes in combination with Bio-Oss for guided bone regeneration. International Journal of Oral \& Maxillofacial Implants. 1997;12(6):844-52.

11. Marx RE. Platelet-rich plasma: evidence to support its use. J Oral Maxillofac Surg. 2004;62:489- 496

12. Marx RE. Platelet-rich plasma (PRP): what is PRP and what is not PRP? Implant Dent. 2001;10:225-228

13. Marx RE. In: Tissue Engineering: Applications in Maxillofacial Surgery and Periodontics. Lynch SE, Genco RJ, Marx RE, editor. Chicago: Quintessence; 1999. Plateletrich plasma: a source of multiple autologous growth factors for bone grafts; pp. 71-82

14. Nikolidakis D, Jansen JA. The biology of platelet-rich plasma and its application in oral surgery: literature review. Tissue Engineering: Part B. 2008;14:249-258

15. Weibrich, G.; Hansen, T.; Kleis, W.; Buch, R.; Hitzler, W. Effect of platelet concentration in platelet-rich plasma on peri-implant bone regeneration. Bone 2004, 34, 665-671

16. Ghanaati, S.; Booms, P.; Orlowska, A.; Kubesch, A.; Lorenz, J.; Rutkowski, J.; Landes, C.; Sader, R.; Kirkpatrick, C.; Choukroun, J. Advanced platelet-rich fibrin: A new concept for cell-based tissue engineering by means of inflammatory cells. J. Oral Implantol. 2014, 40, 679-689.

17. Bielecki, T.; Dohan Ehrenfest, D.M.; Everts, P.A.; Wiczkowski, A. The role of leukocytes from L-PRP/L-PRF in wound healing and immune defense: New perspectives. Curr. Pharm. Biotechnol. 2012, 13, 1153-1162.

18. DelBalso A, Greiner F, Licata M. Role of diagnostic imaging in evaluation of the dental implant patient. Radiographics. 1994;14(4):699-719.
19. Jacobs R, Salmon B, Codari M, Hassan B, Bornstein M. Cone beam computed tomography in implant dentistry: recommendations for clinical use. BMC oral health. 2018;18(1):88.

20. Foster TE, Puskas BL, Mandelbaum BR, Gerhardt MB, Rodeo SA. Platelet-rich plasma: from basic science to clinical applications. The American J Sports Med 2009;37(11):2259-2272.

21. Okuda K, Kawase T, Momose M, Murata M, Saito Y, Suzuki H, Wolff LF, Yoshie H. Platelet-rich plasma contains high levels of platelet-derived growth factor and transforming growth factor $\beta$ and modulates the proliferation of periodontally related cells in vitro. J Periodontol 2003;74(6):849-857.

22. Arpornmaeklong $\mathrm{P}$, Kochel $\mathrm{M}$, Depprich R, Kübler $\mathrm{N}$, Würzler K. Influence of platelet-rich plasma (PRP) on osteogenic differentiation of rat bone marrow stromal cells. An in vitro study. Int J Oral Maxillofac Surg 2004;33(1):60-70.

23. Kawase T, Okuda K, Wolff LF, Yoshie H. Platelet-rich plasmaderived fibrin clot formation stimulates collagen synthesis in periodontal ligament and osteoblastic cells in vitro. J Periodontol 2003;74 (6):858-864.

24. Kanno T, Takahashi T, Tsujisawa T, Ariyoshi W, Nishihara T. Plateletrich plasma enhances human osteoblast-like cell proliferation and differentiation. J Oral Maxillofac Surg 2005;63(3):362-369.

25. He L, Lin Y, Hu X, Zhang Y, Wu H. A comparative study of platelet-rich fibrin (PRF) and platelet-rich plasma (PRP) on the effect of proliferation and differentiation of rat osteoblasts in vitro. Oral Surg Oral Med Oral Pathol Oral Radiol Endod 2009;108 (5):707-713.

26. Anitua E, Prado R, Troya M, Zalduendo M, de la Fuente M, Pino A, Muruzabal F, Orive G. Implementation of a more physiological plasma rich in growth factor (PRGF) protocol: Anticoagulant removal and reduction in activator concentration. Platelets 2016;27(5):459-466.

27. Karukonda SR, Flynn TC, Boh EE, McBurney EI, Russo GG, Millikan LE. The effects of drugs on wound healingpart II. Specific classes of drugs and their effect on healing wounds. Int J Dermatol 2000;39(5):321-333.

28. Kobayashi E, Fluckiger L, Fujioka-Kobayashi M, Sawada K, Sculean A, Schaller B, Miron RJ. Comparative release of growth factors from PRP, PRF, and advanced-PRF. Clin Oral Investig 2016;20(9):2353-2360. 
29. Miron R, Fujioka-Kobayashi M, Bishara M, Zhang Y, Hernandez M, Choukroun J. Platelet Rich Fibrin and Soft Tissue Wound Healing: a Systematic Review. Tissue Eng Part B Rev 2016;23 (1):83-99

30. Fujioka-Kobayashi M, Miron RJ, Hernandez M, Kandalam U, Zhang Y, Choukroun J. Optimized Platelet Rich Fibrin With the Low Speed Concept: Growth Factor Release, Biocompatibility and Cellular Response. J Periodontol 2016:1-17.

31. Anitua E. Plasma rich in grow th factors: Preliminar y results of use in the preparation of future sites for implants. Int J Oral Maxillofac Impl ants 1999;14:529-535.

32. Anitua EA. Enhancement of osseointegration by generating a dynamic implant surface. J O ral Implantol 2006; 32:72-76

33. Zechner W, Tangl S, Tepper G, et al. Influence of plateletrich plasma on osseous healing of dental implants: A histologic an d histomorphometric study in minipigs. Int J Oral Maxillofac Implants 2003;18:15-22.

34. Fontana S, Olmedo DG, Linares JA, et al. Effect of platelet-rich plasma on the peri-implant bone response: An experimental study. Implant Dent 20 04;13:73-78
35. Ergun G, Egilmez F, Cekic-Nagas I, Kar aca IR, Bozkaya $\mathrm{S}$. Effec $\mathrm{t}$ of platelet-rich plasma on the outcome of early loaded dental im- plants: A three-year follow-up study. J Oral Implantol 2013;39:256- 263.

36. He L, Lin Y, Hu X, Zhang Y, Wu H. A comparative study of platelet rich fibrin (PRF) and platelet-rich plasma (PRP) on the effect of proliferation and differentiation of rat osteoblasts in vitro. Oral Surg Oral Med Oral Pathol Oral Radiol Endod 20 09;108:707-713.

37. Anitua E, Orive G, Aguirre JJ, Ardanza B, Andia I. 5-year clinical experience with BTI dent al implants: Risk factors for implant failure. J Clin Periodonto 1 2008;35:724-732 .

38. Monov G, Fuerst G, Tepper G, Watzek G, Zechner W, Watzek G. The effect of platelet-rich plasma upon implant stabilit y measured by resonance frequency analysis in the lower anterior mandibles. Clin Oral Implant s Res 2005;16:461-465.

39. Aparicio C, Lang NP, Rangert B. Validity and clinical significance of biomechanical testing of implant/b one interface. Clin Oral Implants Res 2006;17:2-7. 